\title{
A non-homogeneous vacuum in a holographic model for large N QCD
}

\section{Carlos Alfonso Ballon Bayona*}

Department of Mathematical Sciences, Durham University,

E-mail: c.a.m.ballonbayona@durham.ac.uk

\section{Kasper Peeters}

Department of Mathematical Sciences, Durham University,

E-mail: kasper.peeters@durham.ac.uk

\section{Marija Zamaklar}

Department of Mathematical Sciences, Durham University,

E-mail: marija.zamaklar@durham.ac.uk

\begin{abstract}
We investigate the instability of the homogeneous vacuum of low-temperature QCD at large-N due to a chiral chemical potential. We use the holographic Sakai-Sugimoto model to map large-N QCD, in the non-perturbative regime, to a five dimensional theory. We find at some critical chiral density a new vacuum that breaks translational invariance. This vacuum arises as a consequence of the Chern-Simons coupling present in the five dimensional theory. This presentation is based on our recent publication [1].
\end{abstract}

Sixth International Conference on Quarks and Nuclear Physics April 16-20, 2012

Ecole Polytechnique, Palaiseau, Paris

${ }^{*}$ Speaker. 


\section{Introduction}

One important problem in QCD is the investigation of the ground state at nonzero temperature and density. At finite density there are different possible mechanisms for the quarks or hadrons to condense. For instance, at very high densities perturbative QCD predictions indicate the formation of a color superconducting ground state. This phase is spatially isotropic and homogeneous. However, in the large-N limit there is a competing mechanism suggesting the formation of a nonhomogeneous configuration [2].

We investigate the vacuum of low temperature QCD at large- $\mathrm{N}$ in the presence of a chiral chemical potential [1]. A nonzero chiral density can be interpreted as the imbalance between quarks with different chirality ${ }^{1}$. Using the holographic Sakai-Sugimoto model [3] we map this problem to a five dimensional Maxwell-Chern-Simons theory. As shown in [4, 5], these class of theories may be unstable depending on the Chern-Simons coupling. We find that above a critical chiral density the homogeneous configuration is unstable towards the formation of a spatially modulated phase.

\section{Large-N QCD from holography : The Sakai-Sugimoto model}

Consider the background generated by a stack of N D4-branes in type IIA string theory. It has a metric, Ramond-Ramond four-form and dilaton given by

$$
\begin{aligned}
\mathrm{d} s^{2} & =\frac{u^{3 / 2}}{R^{3 / 2}} \eta_{\mu v} \mathrm{~d} x^{\mu} \mathrm{d} x^{v}+\frac{u^{3 / 2}}{R^{3 / 2}} f(u) \mathrm{d} \tau^{2}+\frac{R^{3 / 2}}{u^{3 / 2}} \frac{\mathrm{d} u^{2}}{f(U)}+R^{3 / 2} u^{1 / 2} \mathrm{~d} \Omega_{4}^{2}, \\
e^{\phi} & =g_{s} \frac{u^{3 / 4}}{R^{3 / 4}}, \quad F_{4}=\frac{\left(2 \pi l_{s}\right)^{3} N}{V_{S^{4}}} \varepsilon_{4}, \quad f(u)=1-\frac{u_{K K}^{3}}{u^{3}},
\end{aligned}
$$

where $\varepsilon_{4}$ is the volume form on $S^{4}$ and $V_{S^{4}}$ its volume. The index $\mu$ defines the directions of the 4-d dual gauge theory, and $\tau$ is an extra coordinate on a circle. The curvature radius is given by $R=\left(\pi g_{s} N\right)^{1 / 3} l_{s}$ where $g_{s}$ is the string coupling and $l_{s}=\sqrt{\alpha^{\prime}}$ is the string length. The period of the compact coordinate introduces a four-dimensional mass scale

$$
\delta \tau=\frac{4 \pi}{3} \frac{R^{3 / 2}}{u_{K K}^{1 / 2}} \equiv \frac{2 \pi}{M_{K K}},
$$

It is useful to introduce a pair of dimensionless coordinates $y$ and $z$ defined by the relations

$$
u=u_{K K}\left(1+y^{2}+z^{2}\right)^{1 / 3} \quad, \quad \tau=\frac{\delta \tau}{2 \pi} \arctan \left(\frac{z}{y}\right) .
$$

Sakai and Sugimoto considered $N_{f}$ D8-D $\overline{\mathrm{D} 8}$ branes in the probe limit $\left(N_{f} \ll N\right)$ [3]. These branes fill out the 4-d spacetime and the 4-sphere and trace out a curve in $(u, \tau)$. In this work we consider the antipodal brane embedding that corresponds to $y=0$. In this case the D 8 branes and $(\overline{\mathrm{D} 8})$ branes are positioned at antipodal points at the boundary $u \rightarrow \infty$ and merge at the infrared cut-off $u=u_{K K}$. The merging of D8 branes and ( $\left.\overline{\mathrm{D} 8}\right)$ branes is a holographic realization of the spontaneous breaking of the $U\left(N_{f}\right)_{L} \times U\left(N_{f}\right)_{R}$ chiral symmetry in large-N QCD. As a consequence, the Sakai-Sugimoto model includes the chiral lagrangian for the pion at low energies.

\footnotetext{
${ }^{1} \mathrm{~A}$ possible source of this imbalance is an instanton configuration.
} 
The induced metric corresponding to the antipodal brane embedding takes the form

$$
d s_{D 8}^{2}=a(z) g_{m n}(z) d x^{m} d x^{n}+b(z) d \Omega_{4}^{2} \equiv G_{M N} d x^{M} d x^{N},
$$

where

$$
\begin{aligned}
g_{m n} d x^{m} d x^{n} & =M_{K K}^{2} K_{z}^{2 / 3} \eta_{\mu v} d x^{\mu} d x^{v}+K_{z}^{-2 / 3} d z^{2} \quad, \quad K_{z} \equiv 1+z^{2}, \\
a(z) & =\frac{8}{27} M_{K K} R^{3} K_{z}^{-1 / 6} \quad, \quad b(z)=\frac{2}{3} M_{K K} R^{3} K_{z}^{1 / 6} .
\end{aligned}
$$

The abelian Dirac-Born-Infeld (DBI) action and the Chern-Simons term corresponding to the D8- $\overline{\mathrm{D} 8}$ brane are given by

$$
\begin{aligned}
S_{D B I} & =-\mu_{8} \int_{D 8} d^{9} x e^{-\phi} \sqrt{-\left|G_{M N}+2 \pi \alpha^{\prime} \mathscr{F}_{M N}\right|} \\
S_{C S} & =\mu_{8} \frac{\left(2 \pi \alpha^{\prime}\right)^{3}}{3 !} \int_{D 8} \mathscr{A} \wedge \mathscr{F}^{2} \wedge F_{4}
\end{aligned}
$$

where $\mathscr{A}$ and $\mathscr{F}_{M N}$ are the one-form and field strength associated with the 9-d gauge field. If we restrict to 5-d gauge fields $\mathscr{A}_{m}$ depending only on the coordinates $x^{m}=\left(x^{\mu}, z\right)$ we can integrate the $S^{4}$ coordinates and obtain

$$
S_{D B I}+S_{C S}=-\int d^{4} x d z \gamma(z) \sqrt{-\left|g_{m n}+\beta(z) \mathscr{F}_{m n}\right|}+\alpha \int_{M^{4} \times \mathbb{R}} \mathscr{A}^{2}
$$

where

$$
\gamma(z)=\frac{\bar{\lambda}^{3} N}{\pi^{2}} K_{z}^{-1 / 3}, \beta(z)=\frac{1}{2 \bar{\lambda}} K_{z}^{1 / 6}, \bar{\lambda}=\frac{\lambda}{27 \pi}, \lambda=g_{Y M}^{2} N=2 \pi M_{K K} g_{s} N l_{s}, \alpha=\frac{N}{24 \pi^{2}} .
$$

In the large $\lambda$ limit the DBI term reduces to the Maxwell action so we obtain

$$
S_{M a x}+S_{C S}=-\frac{\kappa}{2} \int \mathrm{d}^{4} x \mathrm{~d} z \sqrt{-g} \mathscr{F}^{m n} \mathscr{F}_{m n}+\frac{\alpha}{4} \varepsilon^{\ell m n p q} \int \mathrm{d}^{4} x \mathrm{~d} z \mathscr{A}_{\ell} \mathscr{F}_{m n} \mathscr{F}_{p q}
$$

with $\kappa=\bar{\lambda} N /\left(8 \pi^{2}\right)$. In the non-abelian case, the large $\lambda$ limit of the DBI action reduces to a $5-\mathrm{d}$ Yang-Mills action and we obtain

$$
S_{Y M}+S_{C S}=-\frac{\kappa}{2} \int \mathrm{d}^{4} x \mathrm{~d} z \sqrt{-g} \operatorname{tr}\left[\mathscr{F}^{m n} \mathscr{F}_{m n}\right]+\alpha \int_{M^{4} \times \mathbb{R}} \operatorname{tr}\left(\mathscr{A} \mathscr{F}^{2}-\frac{1}{2} \mathscr{A}^{3} \mathscr{F}+\frac{1}{10} \mathscr{A}^{5}\right)
$$

A gauge field fluctuation can be expanded, in the $\mathscr{A}_{z}=0$ gauge, as

$$
\mathscr{A}_{\mu}(x, z)=\hat{\mathscr{V}}_{\mu}(x)+\hat{\mathscr{A}}_{\mu}(x) \psi_{0}(z)+\sum_{n=1}^{\infty}\left[v_{\mu}^{n}(x) \psi_{2 n-1}(z)+a_{\mu}^{n}(x) \psi_{2 n}(z)\right]
$$

where

$$
\begin{aligned}
& \hat{\mathscr{V}}_{\mu}(x)=\frac{1}{2} U^{-1}\left[\mathscr{A}_{\mu}^{L}+\partial_{\mu}\right] U+\frac{1}{2} U\left[\mathscr{A}_{\mu}^{R}+\partial_{\mu}\right] U^{-1}, \\
& \hat{\mathscr{A}}_{\mu}(x)=\frac{1}{2} U^{-1}\left[\mathscr{A}_{\mu}^{L}+\partial_{\mu}\right] U-\frac{1}{2} U\left[\mathscr{A}_{\mu}^{R}+\partial_{\mu}\right] U^{-1}, \quad U(x)=\exp \left(\frac{i \pi(x)}{f_{\pi}}\right),
\end{aligned}
$$


and $\psi_{n}(z)$ are Kaluza-Klein modes in the $z$ direction. Using (2.11) and the Kaluza-Klein equations we get a 4-d lagrangian of mesons and external $U(1)$ fields. The vector (axial vector) mesons are represented by the fields $v_{\mu}^{n}(x)\left(a_{\mu}^{n}(x)\right)$ and the pion is represented by the field $\pi(x)$. In addition, we have external $U(1)$ fields represented by $\mathscr{A}_{\mu}^{L / R}$.

The 4-d effective action arising from the DBI term includes the Skyrme action :

$$
S_{\text {Skyrme }}=\int d^{4} x\left(\frac{f_{\pi}^{2}}{4} \operatorname{tr}\left(L_{\mu}\right)^{2}+\frac{1}{32 e_{S}^{2}} \operatorname{tr}\left[L_{\mu}, L_{v}\right]^{2}\right),
$$

where

$$
L_{\mu} \equiv U^{-1} \partial_{\mu} U \quad, \quad f_{\pi}^{2} \equiv \frac{4}{\pi} \kappa=\frac{1}{54 \pi^{4}} \lambda N_{c}, \quad, \quad e_{S}^{-2} \equiv \kappa \int d z K_{z}^{-1 / 3}\left(1-\psi_{0}^{2}\right)^{2} .
$$

On the other hand, the CS term leads to the WZW action :

$$
S_{W Z W}=-\frac{N_{c}}{48 \pi^{2}} \int_{M^{4}} Z-\frac{N_{c}}{240 \pi^{2}} \int_{M^{4} \times \mathbb{R}} \operatorname{tr}\left(g d g^{-1}\right)^{5}
$$

where $Z$ is a 4-d function of the pion and the $U(1)$ fields and $g$ is a 5-d gauge function that satisfies the boundary conditions $g(x, z \rightarrow-\infty)=1$ and $g(x, z \rightarrow \infty)=U^{-1}(x)$.

\section{Holographic currents and chiral chemical potential}

We can always split the gauge field $\mathscr{A}_{m}$ into a vector field $\mathscr{A}_{m}^{V}$ and axial-vector field $\mathscr{A}_{m}^{A}$

$$
\mathscr{A}_{m}(x, z)=\mathscr{A}_{m}^{V}(x, z)+\mathscr{A}_{m}^{A}(x, z)
$$

The vector and axial-vector fields transform under $z \rightarrow-z$ as

$$
\mathscr{A}_{\mu}^{V / A}(-z, x)= \pm \mathscr{A}_{\mu}^{V / A}(z, x) \quad, \quad \mathscr{A}_{z}^{V / A}(-z, x)=\mp \mathscr{A}_{z}^{V / A}(z, x),
$$

and the $\mu$ component of these fields satisfy the boundary conditions

$$
\mathscr{A}_{\mu}^{V}(z= \pm \infty, x)=A_{\mu}^{V}(x) \quad, \quad \mathscr{A}_{\mu}^{A}(z= \pm \infty, x)=\mp A_{\mu}^{A}(x),
$$

where $A_{\mu}^{V / A}(x)$ are boundary vector and axial-vector gauge fields that couple to 4-d vector and axial currents. Holography relates these currents to the 5-d action :

$$
\left\langle J_{V / A}^{\mu}(x)\right\rangle=\frac{\delta S}{\delta A_{\mu}^{V / A}(x)}= \pm \frac{\delta S}{\delta \mathscr{A}_{\mu}^{V / A}(x, z=\infty)} .
$$

Adding a chiral chemical potential to the 4-d large-N QCD corresponds to adding a source to the chiral density $J_{A}^{0}(x)$, which implies the boundary condition $A_{\mu}^{A}(x)=\mu_{A} \delta_{\mu, 0}$ and $A_{\mu}^{V}(x)=0$. The chiral density $\left\langle J_{A}^{0}\right\rangle$ can be obtained from the holographic map (3.4). 


\section{The non-homogeneous ansatz}

A homogeneous phase corresponds to turning on the $\mathscr{A}_{0}(z)$ component only. In this case the Chern-Simons contribution to the equations of motion vanishes and the solution is trivial :

$$
\mathscr{A}_{0}(z)=\frac{2}{\pi} \mu_{A} \arctan z
$$

If we turn on the transverse part of the gauge field we still can find a non-trivial solution of the form ${ }^{2}$.

$$
\mathscr{A}_{0}=f(z), \quad \overrightarrow{\mathscr{A}}=h(z)\left[\cos \left(k x_{1}\right) \hat{x}_{2}-\sin \left(k x_{1}\right) \hat{x}_{3}\right] .
$$

In this case we obtain the coupled equations

$$
\begin{gathered}
M_{K K}^{2} K_{z} \partial_{z} f=\tilde{\rho}-\frac{3}{2} \frac{\alpha}{\kappa} k h^{2} \\
M_{K K}^{4} K_{z} \partial_{z}\left[K_{z} \partial_{z} h\right]-M_{K K}^{2} K_{z}^{2 / 3} k^{2} h+3 \frac{\alpha}{\kappa} k h\left[\tilde{\rho}-\frac{3}{2} \frac{\alpha}{\kappa} k h^{2}\right]=0
\end{gathered}
$$

where $\tilde{\rho}$ is an integration constant. The axial density is proportional to this constant, i.e. $\left\langle J_{A}^{0}\right\rangle=$ $-4 \kappa \tilde{\rho}$. In addition we get non-zero expectation value for the transverse currents :

$$
\left\langle J_{V}^{2}\right\rangle=-4 \kappa M_{K K}^{2} \lim _{z \rightarrow \infty}\left[K_{z} \partial_{z} h\right] \cos \left(k x_{1}\right) \quad, \quad\left\langle J_{V}^{3}\right\rangle=4 \kappa M_{K K}^{2} \lim _{z \rightarrow \infty}\left[K_{z} \partial_{z} h\right] \sin \left(k x_{1}\right) .
$$

Here we assumed that $h(z)$ is even in $z$. In order to solve numerically this system it is convenient to introduce dimensionless variables defined by

$$
f=\bar{\lambda} M_{K K} \hat{f} \quad h=\bar{\lambda} M_{K K} \hat{h} \quad k=M_{K K} \hat{k} \quad, \quad \tilde{\rho}=\bar{\lambda} M_{K K}^{3} \hat{\rho} .
$$

\section{Analysis of the ground state}

The left panel of Fig. 1 shows the values of $\hat{\rho}$ and $\hat{k}$ for which an instability of the homogeneous phase sets in. The dashed and dotted curves correspond to additional instabilities of axial and vectorial nature respectively, which set in at higher values of the density. This is obtained from an analysis of linear fluctuations of the form (4.2). For values of $\hat{\rho}$ above $\hat{\rho}_{\text {crit }} \approx 2.35$ there is a non-homogeneous phase in a region $\hat{k}_{1}<\hat{k}<\hat{k}_{2}$ corresponding to a solution of the non-linear equations (4.3) and (4.4). The non-homogeneous phase (4.2) has a lower energy than the homogeneous configuration and achieves its minimum energy for $\hat{k}=\hat{k}_{\text {min }}$. We show in the right panel of Fig. 1 that $\hat{k}_{\text {min }}$ is almost independent of the critical chiral density $\hat{\rho}$.

The thick line in the left panel of Fig. 2 shows the chemical potential $\hat{\mu}_{A}$ as a function of the chiral density $\hat{\rho}$ for the non-homogeneous ground state. The thin diagonal dashed line shows the relation $\hat{\mu}_{A}=\pi \hat{\rho}_{A} / 2$ for the homogeneous vacuum. The normalizable solution $\hat{h}(z)$ can be expanded as $\hat{h}(z)=\alpha_{0} / z+\ldots$. The expectaction values of the currents $\left\langle J_{V}^{2}\right\rangle$ and $\left\langle J_{V}^{3}\right\rangle$ are proportional to $\alpha_{0}$. The right panel of Fig. 2 shows $\alpha_{0}$ as a function of $\hat{\rho}$.

It is important to remark that a non-homogeneous ground state has been previously obtained in the deconfined chirally symmetric phase of the Sakai-Sugimoto model at finite quark density [6].

\footnotetext{
${ }^{2}$ This ansatz is motivated by the construction of $[4,5]$ in five dimensional Maxwell-Chern-Simons theories
} 

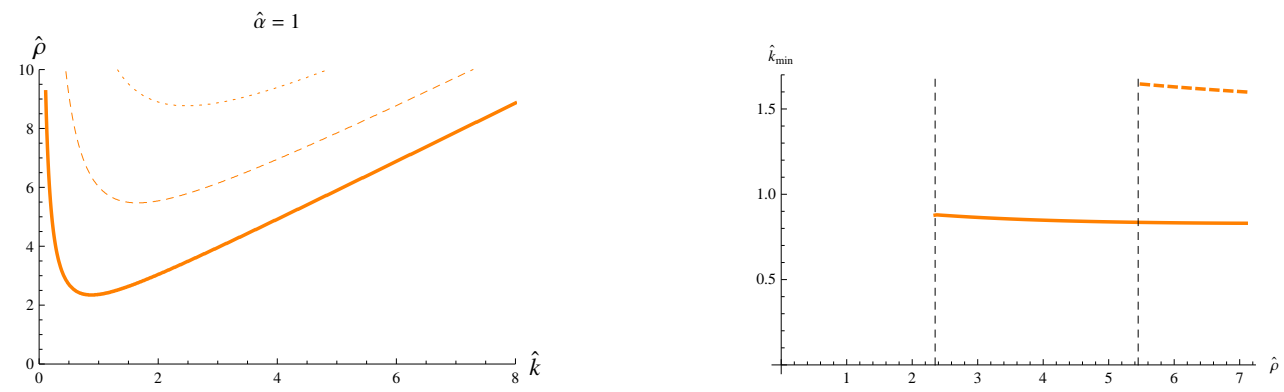

Figure 1: Left: Region in the $\hat{\rho}$ vs $\hat{k}$ plane where an instability sets in. Right: The momentum of the spatial modulation as a function of $\hat{\rho}$ for the non-homogeneous ground state.
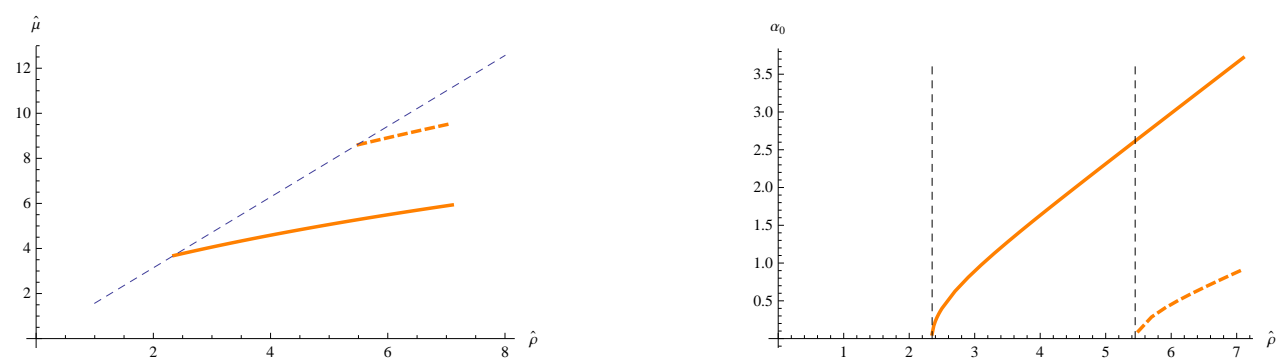

Figure 2: Left: Chiral chemical potential $\hat{\mu}_{A}$ as a function of the chiral density $\hat{\rho}$ for the non-homogeneous ground state. Right: Coefficient $\alpha_{0}$ as a function of $\hat{\rho}$.

\section{References}

[1] C. A. Ballon Bayona, K. Peeters and M. Zamaklar, JHEP 1106, 092 (2011). [arXiv:1104.2291 [hep-th]].

[2] D. V. Deryagin, D. Y. .Grigoriev and V. A. Rubakov, Int. J. Mod. Phys. A 7, 659 (1992).

[3] T. Sakai and S. Sugimoto, Prog. Theor. Phys. 113, 843 (2005) [hep-th/0412141].

[4] S. Nakamura, H. Ooguri and C. -S. Park, Phys. Rev. D 81, 044018 (2010). [arXiv:0911.0679 [hep-th]].

[5] H. Ooguri and C. -S. Park, Phys. Rev. D 82, 126001 (2010).

[6] H. Ooguri and C. -S. Park, Phys. Rev. Lett. 106, 061601 (2011) [arXiv:1011.4144 [hep-th]]. 